\title{
$A$-PROPER MAPPINGS AND THEIR UNIFORM LIMITS 1
}

\author{
BY P. M. FITZPATRICK
}

Communicated by Felix Browder, December 29, 1971

In the following $X$ and $Y$ will always denote real Banach spaces. A quadruple of sequences $\Gamma=\Gamma\left(\left\{X_{n}\right\},\left\{P_{n}\right\},\left\{Y_{n}\right\},\left\{Q_{n}\right\}\right)$ is called a complete projection scheme for mappings from $X$ to $Y$ provided that for each positive integer $n, X_{n}$ and $Y_{n}$ are subspaces of $X$ and $Y$, respectively, of the same finite dimension, and $P_{n}: X \rightarrow X_{n}$ and $Q_{n}: Y \rightarrow Y_{n}$ are bounded linear projections onto $X_{n}$ and $Y_{n}$, respectively, such that $P_{n}(x) \rightarrow x$ and $Q_{n}(y) \rightarrow y$ for each $x \in X$ and $y \in Y$. We shall assume throughout that the Banach spaces considered are equipped with such projection schemes. Given $D \subset X, y \in Y$, and $T: D \subset X \rightarrow Y$, one may attempt to obtain solutions to the equation

$$
T(x)=y, \quad x \in D,
$$

as limits of solutions to the approximating equations

$$
Q_{n} T(x)=Q_{n}(y), \quad x \in D \cap X_{n} .
$$

Petryshyn (see [8] and [9] for references) investigated the class of mappings for which one may implement this constructive approximation approach, and the notion which evolved from these investigations was that of an $A$-proper (approximation proper) mapping.

Definition 1. A mapping $T: D \subset X \rightarrow Y$ is called A-proper with respect to $\Gamma$ provided that whenever $\left\langle n_{k}\right\rangle$ is a sequence of positive integers and $\left\langle x_{n_{k}}\right\rangle$ is a corresponding bounded sequence such that $x_{n_{k}} \in X_{n_{k}} \cap D$, for each $k$, with $Q_{n_{k}} T\left(x_{n_{k}}\right) \rightarrow y$, then $\left\langle x_{n_{k}}\right\rangle$ has a subsequence which converges to $x_{0} \in D$, with $T\left(x_{0}\right)=y$.

Continuous $A$-proper mappings, when defined on closed sets, are proper [9], while continuous proper mappings need not be $A$-proper. Compact displacements are $A$-proper, as are certain classes of strongly monotone and strongly accretive mappings.

In [3] Browder and Petryshyn defined a multivalued topological degree for continuous $A$-proper mappings; this degree has properties analogous to those of the integer valued degree defined for compact displacements by Leray and Schauder. Our first result gives conditions which ensure that the set of solutions of equation (1) is a continuum (i.e., compact,

AMS 1970 subject classifications. Primary 47H10, 47H15; Secondary 39A40.

1 The results reported here are part of a Ph.D. thesis written at Rutgers University under Professor W. V. Petryshyn. 
connected, and nonempty). It is a special case of a more general result which, for simplicity, we do not present here.

THEOREM 1. Let $D \subset X$ be open and bounded, with $T: \bar{D} \subset X \rightarrow Y$ proper and A-proper. Suppose there exists a sequence $\left\{T^{k}: \bar{D} \rightarrow Y\right\}$ of A-proper mappings such that

(i) $\left.Q_{n} T^{k}\right|_{\bar{D} \cap X_{n}}$ is continuous for each $k$ and $n$.

(ii) $\operatorname{Sup}\left\{\left\|T^{k}(x)-T(x)\right\| \mid x \in \bar{D}\right\}=\delta_{k}>0$, and $\delta_{k} \rightarrow 0$ as $k \rightarrow \infty$.

(iii) $T^{k}$ is one-to-one when restricted to $\left(T^{k}\right)^{-1}\left(B\left(0, \delta_{k}\right)\right)$.

Then if $\operatorname{Deg}(T, D, 0) \neq\{0\}, T^{-1}(0)$ is a continuum.

One first shows that if $L$ is $A$-proper with $\operatorname{Deg}(L, D, 0)$ well defined, then for $A$-proper mappings $S$ sufficiently close to $L$,

$$
\operatorname{Deg}(L, D, 0)=\operatorname{Deg}(S, D, 0) .
$$

Then, by means of the weak additivity result for the $A$-proper degree, one shows that the assumption that $T^{-1}(0)$ is disconnected implies $\operatorname{Deg}(T, D, 0)$ $=\{0\}$. As a special case of the above theorem one obtains the results of Krasnoselskii and Sobolevskii [7], when $T=I-C$, where $C$ is compact. In addition this result unifies and extends the results on the structure of sets of fixed points obtained by Deimling [4] and Vidossich [11]. ${ }^{2}$

Although, in general, there is no product theorem available for the $A$-proper degree, a product result for the composition of a linear $A$ proper mapping and a translation enables us to prove the following result on the computation of degree, which generalizes the corresponding result for the Leray-Schauder degree (see [10]).

THEOREM 2. Let $D \subset X$ be bounded and open, and suppose $T: D \subset X \rightarrow Y$ is A-proper, continuous, and has a Fréchet derivative at $x_{0} \in D$ which is one to one and A-proper. Then there is an $r>0$ such that

$$
\operatorname{Deg}\left(T, B\left(x_{0}, r\right), T\left(x_{0}\right)\right)=\operatorname{Deg}\left(T_{x_{0}}^{\prime}, B(0, r), 0\right) .
$$

One may show that a condition slightly weaker than the strong continuity of the derivative suffices to ensure that $T$ is $A$-proper iff each $T_{x}^{\prime}$ is $A$-proper.

When $X$ is a Banach space, with $\Gamma=\Gamma\left(\left\{X_{n}\right\},\left\{P_{n}\right\}\right)$ a complete projection scheme for mappings from $X$ into $X$, such that $\left\|P_{n}\right\|=1$, for all $n$, and $C: \bar{B}(0,1) \subset X \rightarrow X$ is a contraction (i.e., $\|C(x)-C(y)\| \leqq \alpha \cdot\|x-y\|$, for all $x, y \in \bar{B}(0,1)$, for $\alpha \in(0,1)$ fixed), it is unknown whether $I-C: \bar{B}(0,1) \subset X \rightarrow X$ is $A$-proper or not. By imposing various additional hypotheses on $C$ and $X$ one may prove $A$-properness. However, one may prove $A$-properness at certain points. Specifically, call a mapping

2 Details of the results stated here will appear elsewhere. 
$T: D \subset X \rightarrow Y$ A-proper at $y_{0} \in Y$ if Definition 1 is satisfied with $y$ restricted to this particular $y_{0}$.

THEOREM 3. Let $X$ be a reflexive Banach space with $\Gamma\left(\left\{X_{n}\right\},\left\{P_{n}\right\}\right) a$ complete projection scheme for mappings from $X$ into $X$ such that $\left\|P_{n}\right\|=1$, for all $n$. Suppose $F \subset X$ is convex and closed, and $C: F \rightarrow F$ is a contraction. Then whenever $y_{0} \in X$ is such that $C(x)+y_{0} \in F$ for all $x \in F, I-C$ : $F \rightarrow X$ is A-proper at $y_{0}$.

The above result allows one to obtain fixed points of contractions $C: \bar{B}(0,1) \subset X \rightarrow X$ such that $C(B) \subset B$ by projection approximation methods as opposed to iteration. We add that the above theorem may be extended to include the strongly semicontractive mappings examined by Kirk [6], and in this manner we obtain Kirk's fixed point results in [6] by means of projectional approximation methods.

We will now define a multivalued topological degree for certain uniform limits of $A$-proper mappings.

Definition 2. Let $D \subset X$ be open and bounded and suppose $S: \bar{D} \subset X$ $\rightarrow Y$ is bounded. Then, whenever $T: \bar{D} \subset X \rightarrow Y$ is such that $T+\alpha S$ : $\bar{D} \subset X \rightarrow Y$ is A-proper with $\left.Q_{n}(T+\alpha S)\right|_{\bar{D} \cap X_{n}}$ continuous for $\alpha>0$ and all $n$, and $y \in Y$ is such that $\|T(x)-y\| \geqq C>0$, for all $x \in D$, there exists $\gamma>0$ with $\operatorname{Deg}(T+\alpha S, D, y)$ independent of $\alpha \in(0, \gamma)$. We denote this constant degree by $\operatorname{Deg}_{S}(T, D, y)$, the degree of $T$ on $D$ over $y$ with respect to $S$.

Using the above definition of degree for mappings $T$ as above, such mappings we call $A$-proper with respect to $S$, and utilizing slight refinements of properties of the Browder-Petryshyn degree, we proved, in [5], that this degree enjoyed the properties necessary to be useful. In order to conclude that there exists $x \in D$ with $T(x)=y$ whenever $\operatorname{Deg}_{s}(T, D, y)$ $\neq\{0\}$, one needs to assume $T(\bar{D})$ is closed. In fact even when $T$ is $A$-proper with each $\left.Q_{n} T\right|_{\bar{D}_{n}}$ continuous it does not follow that $T(\bar{D})$ is closed.

In case $D \subset X$, where $D$ is convex and $X$ is reflexive, with $T: \bar{D} \subset X$ $\rightarrow X$ accretive and continuous, and one also assumes either $T$ is weakly continuous or $X$ is equipped with a weakly continuous duality mapping, then $T$ is $A$-proper with respect to $I$. Using the above result one may use the generalized degree to obtain invariance of domain theorems for accretive mappings in addition to fixed point theorems for nonexpansive mappings. Mappings formed by the interwining of accretive and compactness assumptions may also be considered in this framework.

As has been shown in [5], when $X$ is reflexive and $\Gamma=\Gamma\left(\left\{X_{n}\right\},\left\{P_{n}\right\}\right)$ is such that $\left\|P_{n}\right\|=1$, and $P_{n} P_{m}=P_{n}$, for $m \geqq n$, then $\Gamma$ generates a complete projection scheme for mappings from $X$ into $X^{*}$ for which mappings $T: \bar{D} \subset X \rightarrow X^{*}$, where $D$ is convex and $T$ is pseudomonotone and demicontinuous, are $A$-proper with respect to suitable duality mappings. 
Definition 3. Let $D \subset X$, with $S: D \times D \rightarrow X^{*}$. Suppose

(i) for each $x \in D, S(x, \cdot): D \rightarrow X^{*}$ is monotone and

(ii) for each $x \in D, S(\cdot, x): D \rightarrow X^{*}$ is compact,

then $T: D \rightarrow X^{*}$ defined by $T(x)=S(x, x)$, for $x \in D$, will be called nearly monotone.

THEOREM 4. Let $X$ be reflexive with $D \subset X$ open, bounded, and convex. Suppose $H=H(t, x):[0,1] \times \bar{D} \rightarrow X^{*}$ satisfies the following conditions:

(i) $H$ is continuous in $t$, uniformly with respect to $x \in D$.

(ii) $H(0, \cdot)$ is monotone and $H(t, \cdot)$ is nearly monotone for $0<t \leqq 1$.

(iii) For each $t \in[0,1], H(t, \cdot)$ is bounded and demicontinuous and there exists $C_{t}>0$ such that $\|H(t, x)\| \geqq C_{t}>0$, for all $x \in \dot{D}$.

(iv) $H(1, \cdot)(D)$ is closed.

Then whenever the equation $H(0, x)=0, x \in D$ has a solution, the equation $H(1, x)=0, x \in D$, has a solution.

One proves the above result by showing nearly monotone mappings are $A$-proper with respect to certain duality mappings and then showing that the existence of solutions involving monotone mappings ensures the nonvanishing of certain degrees.

For mappings of subsets of $X$ into $X^{*}$ the above continuation principle contains that of Browder [1], [2], where it is assumed the mappings involved are defined on all of $X$, the constants $C_{t}$ are independent of $t \in[0,1]$, and $X^{*}$ is locally uniformly convex.

\section{BIBLIOGRAPHY}

1. F. E. Browder, Mapping theorems for noncompact nonlinear operators in Banach spaces, Proc. Nat. Acad. Sci. U.S.A. 54 (1965), 337-342.

2. 162 (1965/66), 280-283. MR 32 \#8187.

3. F. E. Browder and W. V. Petryshyn, Approximation methods and the generalized topological degree for nonlinear mappings in Banach spaces, J. Functional Analysis 3 (1969), 217-245. MR 39 \#6126.

4. K. Deimling, Fixed points of generalized P-compact operators, Math. Z. 115 (1970), 188-196. MR 41 \#9073.

5. P. M. Fitzpatrick, A generalized degree for the uniform limits of A-proper mappings, J. Math. Anal. Appl. 35 (1971), 159-173.

6. W. A. Kirk, On nonlinear mappings of strongly semicontractive type, J. Math. Anal. Appl. 27 (1969), 409-412. MR 39 \#6128.

7. M. A. Krasnosel'skii and P. E. Sobolevskii, The structure of the set of solutions of equations of parabolic type, Ukrain. Mat. Ž. 16(1964), 319-333; English transl., Amer. Math. Soc. Transl. (2) 51 (1966), 113-131. MR 29 \# 3763.

8. W. V. Petryshyn, Nonlinear equations involving noncompact operators, Proc. Sympos. Pure Math., vol. 18, part 1, Amer. Math. Soc., Providence, R. I., 1970, pp. 206-233. MR 42 \# 1970.

9. - Invariance of domain theorem for locally A-proper mappings and its implications, J. Functional Analysis 5 (1970), 137-159. MR 42 \#914.

10. M. M. Vainberg, Variational methods for the study of nonlinear operators, GITTL, Moscow, 1956; English transl., Holden-Day, San Francisco, Calif., 1964. MR 19, 567; MR 31 \#638.

11. G. Vidossich, On Peano phenomenon, Boll. Un. Mat. Ital. (4) 3 (1970), 33-42. MR 42 \#6674.

Department of Mathematics, Rutgers University, The State University of New JERSEY, NEW BRUNSWICK, NEW JERSEY 08903 